Z-LASER

KOMPAKTE LASERMODULE ZM12 MIT GRÖSSERER OPTIKVIELFALT

Die kleinen industrietauglichen Lasermodule ZM12 von Z-Laser in Freiburg sind speziell als Lösung für OEMs entwickelt worden, die in ihrem Herstellungsprozess zum Beispiel zur Lasermarkierung oder auch bei Bildverarbeitungsanwendungen auf PremiumQualität und verlässliche Leistung setzen. Die ZM12-Produktreihe wird ab sofort mit deutlich größerer Optikvielfalt angeboten. Durch den neu hinzugekommenen Optikkopf und einen optionalen, leicht zu bedienenden Fokusring kann der Nutzer den Laser noch einfacher an seine Bedürfnisse anpassen. „Durch das gute Preis-Leistungs-Verhältnis und das kompakte M12-Schraubengewinde des eigentlichen La- sers ist er ein idealer Einstieg für diverse Anwendungen von Positionierung bis Bildverarbeitung und in sämtlichen Industrien einsetzbar", heißt es. Für raue Umgebungen sei das Gerät durch IP67 vor Staub und Wasser geschützt.

Das Basismodell inklusive Optikkopf ist 85 mm lang und hat einen Durchmesser von 15 $\mathrm{mm}$. Mit dem Fokusring lässt sich laut Z-Laser die Laserprojektion noch einfacher scharf stellen. Als Modell mit integriertem Kabelanschluss (ZM12DM5) reduziere sich die Länge auf nur noch $63 \mathrm{~mm}$. Je nach Ausstattung seien die Module mit roten und infraroten Wellenlängen, einer optischen Ausgangsleistung von bis zu $100 \mathrm{~mW}$ sowie Modulationsfre- quenzen von bis zu 100 kHz erhältlich. Diese Eigenschaften ermöglichten eine einfache Synchronisierung mit einem Kamerasystem oder eine Intensitätseinstellung des Lasers über eine PWM (Puls-Weiten-Modulation). Die Hauptmerkmale der Lasermodule: 5-30VDC Versorgungsspannung über M12Stecker oder 4-6 VDC mit integriertem Kabel, Optische Leistung bis zu $100 \mathrm{~mW}$, Rote und infrarote Wellenlängen erhältlich ( $635 \mathrm{~nm}-850$ nm), TTL Modulation bis 100 kHz, Gauß-/homogene Linienprojektion und weitere Optiken, Festfokus oder manuell fokussierbar und ein kompaktes M12 Schraubengewinde für einfache und vielseitige Befestigung.

CORETECH SYSTEM

\title{
Moldex3D für die CAE-Analyse zur Optimierung von
}

\section{Heißkanalsystemen}

Mold-Masters Ltd., nach eigenen Angaben eines der weltweit führenden Technologieunternehmen für patentierte Innovationen, hat sich für die Software Moldex3D der Taiwanesischen CoreTech System Co. Ltd. als globales und alleiniges CAE-Analyse-Softwarepaket zur Design-Verifizierung und Optimierung von Heißkanalsystemen entschieden. MoldMasters entwickelte unter anderem zahlreiche neue Einsatzmethoden innovativer Heißkanaltechnik, mit deren Hilfe die Herstellung von Spritzgussbauteilen optimiert werden kann.

Um die technische Führerschaft in dem äußerst anspruchsvollen Markt halten zu können, habe Mold-Masters in ein umfassendes Produkt-Management investiert. Ziel sei es, globale CAD/CAM/CAE-Standards für alle Unternehmensbereiche zu schaffen und damit den steigenden Anforderungen im Heißkanaltechnikmarkt gewachsen zu sein.
Mit Moldex3D ist der Anwender laut Mold-Masters in der Lage, Heißkanalkomponenten einfach und präzise zu analysieren, zu verifizieren und hinsichtlich der geforderten Kriterien zu optimieren. So könnten beispielsweise Qualitätsmerkmale des finalen Bauteils, wie die Vermeidung von Füllmustern, Bindenähten oder hochglänzende Oberflächen, garantiert, aber auch wirtschaftliche Aspekte, wie die Reduzierung der Zykluszeit,
Schließkraft beziehungsweise des Einspritzdruckes oder Rohstoff-Einsparungen, realisiert werden.

Moldex3D biete nicht nur realistische Simulationsergebnisse der Formfüllung, eine Bedingung, um als Standard für die Konstruktion und Analyse von Heißkanalsystemen eingesetzt zu werden, sondern außerdem erweiterte 3D-Funktionalitäten, die tiefenanalytische Forschungen ermöglichen.

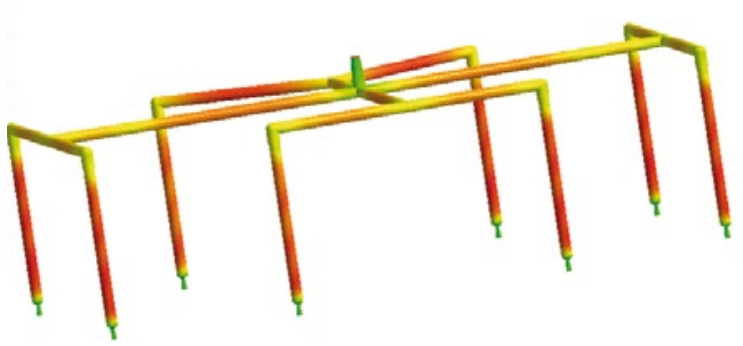

